\title{
Relatos de puérperas sobre vivências de violência obstétrica: Um estudo qualitativo
}

\author{
Postpartum women's reports on experiences of obstetric violence: A qualitative study \\ Informes de mujeres posparto sobre experiencias de violencia obstétrica: Un estudio cualitativo
}

Recebido: 11/06/2021 | Revisado: 19/06/2021 | Aceito: 21/06/2021 | Publicado: 07/07/2021

\author{
Janinne Santos de Melo \\ ORCID: https://orcid.org/0000-0001-8049-6891 \\ Centro Universitário CESMAC, Brasil \\ E-mail: nynne_mello@hotmail.com \\ Evanisa Helena Maio de Brum \\ ORCID: https://orcid.org/0000-0003-0128-591X \\ Centro Universitário CESMAC, Brasil \\ E-mail: evanisa.brum@gmail.com \\ John Victor dos Santos Silva \\ ORCID: https://orcid.org/0000-0003-4671-102X \\ Universidade de São Paulo, Brasil \\ E-mail: john.setedejulho@ gmail.com \\ Camila Maria Beder Ribeiro Girish Panjwani \\ ORCID: https://orcid.org/0000-0003-0203-3079 \\ Centro Universitário CESMAC, Brasil \\ E-mail: cami.beder@gmail.com \\ Sonia Maria Soares Ferreira \\ ORCID: https://orcid.org/0000-0002-4825-171X \\ Centro Universitário CESMAC, Brasil \\ E-mail: sonia.ferreira@cesmac.edu.br \\ Kevan Guilherme Nóbrega Barbosa \\ ORCID: https://orcid.org/0000-0002-9410-7356 \\ Centro Universitário CESMAC, Brasil \\ E-mail: kevanguilherme@gmail.com \\ Mara Cristina Ribeiro \\ ORCID: https://orcid.org/0000-0001-6963-8158 \\ Centro Universitário CESMAC, Brasil \\ E-mail: maracrisribeiro@gmail.com
}

\begin{abstract}
Resumo
Objetivo: Conhecer as vivências de violência obstétrica entre puérperas. Metodologia: Estudo qualitativo realizado por meio de 12 entrevistas com mulheres internadas em duas maternidades de risco habitual na capital de Alagoas, Maceió. Resultado: $\mathrm{Na}$ análise dos relatos emergiram diferentes fatores críticos, tais como: dificuldade de acesso às maternidades, falta de acompanhamento profissional às pacientes, horizontalização do parto, prioridade dos desejos do profissional médico contrários aos maternos, violência verbal, falta de humanização, falta de percepção sobre a violência vivenciada, entre outros. Considerações Finais: As vivências relatadas indicaram diversas formas de violência obstétrica, além do uso de práticas obstétricas consideradas prejudiciais, ineficazes, sem evidências científicas ou realizadas de forma inadequada. O estudo aponta que existem falhas significativas nos processos de cuidado na assistência à mulher, evidenciando o desrespeito aos seus direitos.
\end{abstract}

Palavras-chave: Parto obstétrico; Trabalho de parto; Parto humanizado; Violência contra a mulher; Saúde da mulher.

\begin{abstract}
Objective: To know the experiences of obstetric violence among postpartum women. Methodology: Qualitative study conducted through 12 interviews with women hospitalized in two maternity hospitals at usual risk in the capital of Alagoas, Maceió. Result: In the analysis of the reports, different critical factors emerged, such as: difficulty in accessing maternity hospitals, lack of professional follow-up to patients, horizontalization of childbirth, priority of medical professionals' wishes contrary to maternal ones, verbal violence, lack of humanization, lack of perception of the violence experienced, among others. Final Considerations: The experiences reported indicated several forms of obstetric violence, in addition to the use of obstetric practices considered harmful, ineffective, without scientific evidence or performed inappropriately. The study points out that there are significant flaws in the care processes in assisting women, evidencing the disrespect for their rights.
\end{abstract}

Keywords: Delivery obstetric; Labor obstetric; Humanizing delivery; Violence against women; Women's health. 


\begin{abstract}
Resumen
Objetivo: Conocer las experiencias de violencia obstétrica entre mujeres posparto. Metodología: Estudio cualitativo realizado a través de 12 entrevistas a mujeres hospitalizadas en dos maternidades de riesgo habitual en la capital de Alagoas, Maceió. Resultado: En el análisis de los informes surgieron diferentes factores críticos, tales como: dificultad para acceder a las maternidades, falta de seguimiento profesional de los pacientes, horizontalización del parto, prioridad de los deseos de los profesionales médicos contrarios a los maternos, violencia verbal, falta de humanización, falta de percepción de la violencia vivida, entre otros. Consideraciones finales: Las experiencias reportadas indicaron varias formas de violencia obstétrica, además del uso de prácticas obstétricas consideradas nocivas, ineficaces, sin evidencia científica o realizadas de manera inapropiada. El estudio señala que existen fallas significativas en los procesos de atención en la atención a las mujeres, evidenciando la falta de respeto a sus derechos.

Palabras clave: Parto obstétrico; Trabajo de parto; Parto humanizado; Violencia contra las mujeres; Salud dela mujer.
\end{abstract}

\title{
1. Introdução
}

A violência obstétrica (VO) tem se tornado um problema social grave, com estatísticas alarmantes, no Brasil e no mundo. Ela é caracterizada pela apropriação do corpo e dos processos reprodutivos das mulheres por profissionais de saúde, que muitas vezes são expressos por uso de medicalização de forma indiscriminada, inversão dos processos naturais em patológicos, relações abusivas e coercivas (Rocha \& Ferreira, 2020; Lansky et al, 2019; Sena \& Tesser, 2017), resultando na perda da autonomia e da tomada de decisões dessas mulheres, bem como sobre suas escolhas quanto ao seu corpo e sexualidade (Tesser, Knobel, Andrezzo \& Diniz, 2015; Diniz et al, 2015).

O parto vaginal tornou-se, ao longo dos anos, uma prática desvalorizada, em contrapartida, existe um aumento pela procura de intervenções cirúrgicas desnecessárias, resultado da falta de acesso da população feminina às práticas de educação em saúde. O desconhecimento de seus direitos sexuais e reprodutivos possibilita a prática desumanizada de profissionais de saúde, que acarreta atitudes violentas contra as mulheres (Giacomini \& Hirsch, 2020; Castro, 2020).

Diferentes pesquisas internacionais (Bohren et al, 2014; Chadwick, Cooper \& Harries, 2014; Moyer, Adongo, Aborigo, Hodgson \& Engmann, 2014) têm indicado que muitas mulheres, globalmente, sofrem tratamento inadequado durante o parto, incluindo cuidados abusivos, negligentes ou desrespeitosos. Estudos realizados no Brasil também apontam a ocorrência de situações de violência contra a mulher durante as etapas que envolvem o processo gravídico-puerperal (Katz, Amorim, Giordano, Bastos \& Brilhante, 2020; Gabriel \& Santos, 2020; Sampaio, Tavares \& Herculano, 2019; Biscegli, Grio, Melles, Ribeiro \& Gonsaga, 2015).

A Organização Mundial da Saúde (OMS) contrapõe-se a essa prática ao apontar, em relatório publicado em 2014, que as mulheres têm sido assistidas de forma violenta durante as diferentes etapas que envolvem o parto, passando por vivências de maus-tratos, desrespeito, abusos, negligências e violação dos direitos humanos por profissionais de saúde. Diante dessa realidade, no relatório é preconizado o parto normal da forma mais fisiológica possível, sem induções, com direito à privacidade da mulher, respeito à livre escolha do local e posições para parir, além do apoio indispensável dos profissionais de saúde (OMS, 2014). Outros aspectos indicados como importantes são: o respeito à lei do acompanhante, o estímulo às posições não supinas, bem como a ausência de restrição hídrica (OMS, 1996).

O resgate a uma assistência menos intervencionista, baseada em evidências científicas, que minimize os riscos tanto maternos como fetais e perinatais se caracteriza como proposta recentemente inserida no Brasil, impulsionada a partir de orientações para uma assistência mais humanizada. Dentro desta perspectiva, nas últimas duas décadas, o Brasil vem adotando políticas que estejam em consonância com ações, defesas e protagonismos que dialoguem com essa importante questão pertencente não só ao campo da saúde pública, mas também ancorada no direito da mulher, com o objetivo de garantir cuidados seguros, oportunos e respeitosos em todas as etapas do parto (Brasil, 2003, 2004).

Diante da realidade descrita e da contemporaneidade do tema, torna-se relevante o aprofundamento dos conhecimentos voltados à violência obstétrica, por se tratar de um assunto explorado no Brasil de forma insuficiente, com 
poucos estudos qualitativos de campo que contemplem as mulheres assistidas nos serviços (Jardim \& Modena, 2018).

Esta pesquisa, portanto, teve como objetivo conhecer as vivências de violência obstétrica entre puérperas, tendo como referenciais teóricos as recomendações da OMS (1996) para boas práticas de atenção ao parto e nascimento, a Política Nacional de Humanização (Brasil, 2003) e a Política Nacional de Atenção Integral à Saúde da Mulher (Brasil, 2004).

\section{Metodologia}

Trata-se de pesquisa exploratória, transversal, de abordagem qualitativa. A produção dos dados foi realizada em duas maternidades de Maceió que prestam assistência por meio do Sistema Único de Saúde (SUS) às pacientes da capital e do interior do estado de Alagoas, que estão em trabalho de parto ativo e são classificadas como de risco habitual.

A obtenção das informações nas maternidades ocorreu no período entre 01 de junho de 2018 a 16 de janeiro de 2019, logo após a aprovação do projeto pelo Comitê de Ética em Pesquisa (Parecer nº 2.590.636) em 10 de abril de 2018.

As participantes do estudo foram mulheres em seu puerpério fisiológico, internadas nas duas maternidades, que estivessem nas enfermarias após uma hora do pós-parto vaginal ou cesáreo, podendo este tempo se estender até 24 horas de puerpério. Foram incluídas mulheres maiores de 18 anos, que tiveram partos vaginais ou cesáreos, assistidas por profissional médico obstetra ou enfermeiro obstetra nas maternidades referidas.

A pesquisa foi realizada por meio de entrevistas individualizadas, semiestruturadas, norteadas por um roteiro contendo perguntas abertas que exploravam todo o processo vivido na maternidade (pré-parto, parto e pós-parto). Participaram como voluntárias do estudo 12 mulheres, 6 de cada maternidade. Todas as entrevistas foram gravadas com a autorização das participantes e posteriormente transcritas. Após a transcrição das falas, o conteúdo foi analisado cumprindo-se três fases: préanálise, exploração do material e tratamento e interpretação dos resultados, seguindo a metodologia de Análise de Conteúdo preconizada por Bardin (2011), na modalidade Análise Categorial.

\section{Resultados e Discussão}

$\mathrm{Na}$ análise das falas foram identificados alguns relatos de violência obstétrica propriamente dita, em outros, o uso de práticas obstétricas consideradas contrárias às recomendações da OMS e às políticas nacionais de humanização e de atenção ao parto. Estas situações, emergidas por meio das entrevistas, serão discutidas a seguir.

\section{Dificuldade de acesso, falta de acolhimento e orientação}

A falta de vagas foi indicada como um problema constante, fazendo com que as entrevistadas peregrinassem à procura de outras maternidades; outras faltas como a de acolhimento na admissão e de orientação no pré-natal quanto à maternidade de referência também apareceram como relatos.

A peregrinação da mulher em busca de uma vaga na rede pública de saúde pode ser considerada como violência obstétrica, por estar diretamente relacionada ao seu processo reprodutivo e à anulação dos seus direitos (Rodrigues et al, 2014).

Destaca-se o desgaste físico e a angústia dessa peregrinação.

[...] Quando eu comecei a sentir dor eram umas seis horas da noite da quarta-feira. Quando foi umas dez horas, as dores aumentaram, aí fiquei rodando nas maternidades porque não tinham vagas... Fiquei esperando mais de uma hora lá, não falaram nada e eu sentindo dor! Quando foi depois, disseram que não tinha mais vagas, aí eu fui pra outra maternidade que também não tinha vagas e só de lá que me encaminharam pra cá. (P1)

[...] O posto da minha cidade me mandou um encaminhamento para a maternidade que era a referência do meu município. Quando eu cheguei lá, eles falaram que eu era obesa e a maternidade não tinha maca que suportasse meu peso, nem estrutura para eu ter o bebê lá. Minha pressão estava um pouco alta também. E aí me encaminharam para a 
maternidade de alto risco. Só que quando eu cheguei lá, eles me colocaram de lado e minha pressão baixou. Falaram que eu não tinha peso pra ser de alto risco, acharam um absurdo eu ter sido encaminhada pra lá e eu sentindo dor, né! Foi quando me encaminharam pra essa maternidade, de baixo risco novamente, pois eu não tinha problema nenhum que fosse de alto risco. (P8)

Ofertar informações claras, numa linguagem acessível e de fácil entendimento sobre a assistência prestada é de responsabilidade da equipe de saúde (Andrade, Silva, Diniz \& Caminha, 2016). O estudo evidencia que a falta de orientação prévia, aliada à falta de estrutura e acolhimento das maternidades e seus profissionais, provocam sofrimentos e desconfortos desnecessários. Infere-se que a dificuldade de acesso às maternidades poderia ser evitada se, no acompanhamento pré-natal, essas questões já fossem esclarecidas e organizadas pela equipe de saúde.

Muitas gestantes indicaram a sensação de abandono no pré-parto, a falta de orientação nas dúvidas, nas posições e exercícios facilitadores, além da falta de acompanhamento no trabalho de parto em si:

[...] o tempo que passei lá não chegou ninguém para me orientar! (P.10)

A falta de pessoal para esclarecimentos, orientações e cuidados, além de se caracterizar como um fator estressor, ainda pode comprometer a saúde tanto da mulher quanto do bebê que está por nascer. Portanto, uma equipe treinada e presente pode minimizar riscos e contribuir para que o momento seja de menos tensão.

\section{A violência obstétrica no pré-parto e trabalho de parto}

No ambiente do pré-parto, as falas demostraram que grande parte das pacientes entrevistadas permaneceu horas de seus trabalhos de parto sentindo dor, deitadas, com pouca ou nenhuma utilização dos métodos não farmacológicos para o alívio da dor, sendo auxiliadas por seus acompanhantes e sem nenhum auxílio por parte dos profissionais.

[...] ninguém me perguntou a respeito se estava doendo, se não estava doendo! Era só colocando o dedo e vendo que estava dilatando, aumentando o soro, aumenta, aumenta. Aumentando obviamente aumentava a dor, mas ninguém me perguntou se eu queria sentir dor, se eu achava que estava pronta, como também não perguntou a nenhuma das outras. (P6)

Algumas gestantes relataram que nenhuma ou poucas vezes lhes foi ofertado água ou alimento durante seu trabalho de parto, sem ao menos ser explicado o motivo dessa restrição. Essas parturientes permaneceram longas horas em trabalho de parto e necessitavam de energia suficiente para concluir seus partos:

[...] eu fiquei a madrugada toda sem comer ou beber água e sentindo dor. (P.8)

A restrição hídrica e alimentar dessas parturientes pode estar relacionada à resistência dos profissionais de saúde, mesmo que as evidências científicas mostrem efeitos deletérios do jejum no trabalho de parto (Porto, Amorim \& Souza, 2010).

Outra prática comum encontrada foi a realização de toques vaginais, repetidas vezes, sem explicação sobre o motivo de tal procedimento às gestantes:

[...] a doutora vinha de vez em quando me ver e fazia muitos toques, pra ver se já dava pra o bebê passar, né? (P.3)

[...] fiquei sentindo contrações e fazendo toque, fazendo toque, fazendo toque...(P1) 
O toque vaginal, principalmente após a ruptura das membranas amnióticas, pode ser um fator de risco associado à infecção (Agência Nacional de Vigilância Sanitária [ANVISA], 2017). Apesar do parto vaginal ter menor risco de infecção se comparado ao parto cesáreo, deve-se também evitar a realização excessiva de toques vaginais. Infelizmente, esta prática que é considerada inapropriada, é frequente, além disso, os profissionais a realizam sem nenhum tipo de explicação, o que indica a desapropriação da mulher sobre o seu corpo pois, normalmente, não há espaço para questionamentos pelas mulheres sobre o ato.

\section{Parto e violência}

A OMS (1996) incentiva as práticas que orientam o parto normal, destacando a importância da liberdade de posição e movimento durante o trabalho de parto e o estímulo na adoção de posturas não supinas. No entanto, as gestantes devem adotar a posição que thes for mais confortável.

Foi possível detectar que algumas puérperas entrevistadas, por não saberem ou não serem estimuladas a adotar posições que favorecem o parto, simplesmente ficavam deitadas, andavam ou se sentavam.

[...] a mulher me deu um panfleto só com as informações da maternidade. Depois disse que era pra eu ficar deitada na cama, porque eu já estava em trabalho de parto e o bebê poderia cair no chão! (P9)

Muitas adotavam a posição supina por falta de orientação profissional, desfavorecendo a gravidade, propiciando o aumento de tempo do período expulsivo e a diminuição dos diâmetros pélvicos, se comparados às posições verticalizadas (Schettini, Griboski \& Faustino, 2017).

$\mathrm{Na}$ descrição do momento do parto, as falas evidenciaram a falta de uma relação mais dialogada e a prevalência da autoridade médica na cena, fazendo com que as mulheres tenham seus desejos ofuscados mediante a relação de poder estipulada.

[...] no que a cabeça estava quase saindo, foi que ela [médica] saiu me levando, me arrastando para a sala de parto mesmo. Não podia fazer o parto lá na cama que eu estava não, por causa das complicações. Eu tive tipo um trauma, porque eu coloquei na cabeça que seria cesáreo, aí eu sofri muito. (P3)

Destaca-se que as regras institucionais são importantes para a organização dos serviços de saúde, no entanto, muitos profissionais se utilizam disso para impor vontades e necessidades próprias em detrimento das necessidades das parturientes; acabam por prestar uma assistência obstétrica que desfavorece a autonomia, o respeito e a dignidade da mulher (Assis, 2018).

No Brasil, principalmente nas maternidades públicas, a mulher não tem muito poder para decidir o tipo de parto de sua preferência, na maioria das vezes essa opção fica por parte do profissional médico assistente. A pesquisa identifica a fragilidade da mulher sobre a escolha da via de parto:

[...] cesárea! Você não pode escolher! Você tem que ficar reta e esperar que a cirurgia seja feita. (P.6).

A fala acima indica a imposição de uma escolha que desapropria a mulher do seu direito de vivenciar o parto da forma como ela deseja, retirando-a do processo decisório. Sua participação nas decisões sobre a via de parto e sobre seu próprio corpo está relacionada ao conhecimento que elas têm sobre a fisiologia do parto e puerpério (Reis, Padoin, Toebe, Pasa \& Quadros, 2017). Quando não lhe é ofertado espaços de diálogos e apresentação de alternativas, a mulher é privada de conhecimentos que deveriam ser compartilhados. Como recurso para melhorar essa realidade, é possível indicar práticas extra- 
hospitalares, como o pré-natal, participação em grupos de gestantes e puérperas, que podem favorecer o protagonismo e a autonomia nesse momento de suas vidas.

É evidente que algumas ações podem ser justificadas pela necessidade de preservar a saúde da mãe e do bebê, no entanto, ainda assim, estas precisariam ser esclarecidas à mãe, para que não fossem sentidas ou interpretadas como uma violência. Quando são executadas sem a justificativa adequada, perde-se a oportunidade de a mãe participar de forma ativa das decisões sobre o seu corpo e sobre o processo vivenciado.

\section{Falta de humanização e violência verbal}

Algumas falas fizeram referência à falta de humanização dos profissionais nas duas maternidades, nos relatos pôde-se perceber a vitimização dessas mulheres e o descaso nos processos do cuidar, que muitas vezes não trazem um desfecho favorável ao parto, seja ele normal ou cesáreo. Nota-se a necessidade de uma revisão dos conceitos de parto humanizado por parte dos profissionais de saúde, visto que muitos dos direitos das mulheres são violados.

[...] a humanização é importante, porque se tivesse tratado a gente melhor não estava esse clima; pessoas xingando, gritando e a gente divide o quarto com várias pessoas. [...] É o momento que a gente mais precisa do médico. Eles deveriam tratar a gente com mais carinho, né? (P6)

[...] Eu já estava com a vista embaçada, fraca; o soro que elas colocaram já tinha saído da veia, não desceu, meu braço estava todo inchado. Dessa vez me maltrataram! Elas foram dormir e me deixaram lá gritando e sentindo dor. Parece que quanto mais a gente grita, mais elas deixam sofrer! (P9)

A humanização ganha um patamar ainda mais ético quando indica a necessidade de retirada do papel passivo da parturiente em que alguns profissionais da saúde a obrigam cumprir. Reconhecer os limites da autoridade profissional sobre as decisões e sobre os procedimentos técnicos requer o compromisso do profissional da saúde com a Política Nacional de Humanização e a Política Nacional de Atenção Integral à Saúde da Mulher (Brasil, 2003, 2004). Afinal, são essas que regem as ações de assistência ao parto no SUS.

[...] O que eles fizeram comigo eu não achei certo não! Me deixaram sofrer e quando eu gritava, mandavam eu calar a boca pra que elas dormissem. Maternidade é o lugar pra que a gente seja atendida e não pra que elas estejam dormindo! (P9)

Todos os relatos acima demonstram marcas da violência vivenciada em um momento que, ao contrário, deveria ser de alegria ou, no mínimo, de respeito. A falta de escuta, de acolhimento, de relações mais horizontalizadas, de orientações mais claras, acabam por estressar essas mulheres, favorecendo complicações desnecessárias.

Assim como acontece com a violência física, a violência verbal também causa danos psicológicos irreparáveis e brutais. Na atenção ao parto, muitas mulheres sofrem esse tipo de violência, no entanto, por estarem focadas no nascimento de seus filhos ou filhas, as vítimas podem não se abalar tanto com a violência vivenciada no momento em que a sofrem (Oliveira, Costa, Monte, Veras \& Sá, 2017).

Infelizmente, existe uma banalização da violência, pois as mulheres consideram, muitas vezes, situações de violência como situações normais e até esperam ouvir comentários irônicos ou depreciativos nas maternidades públicas (Aguiar \& D'Oliveira, 2011).

Processos de educação em saúde nos territórios poderiam melhorar essa situação, afinal, o empoderamento feminino é uma estratégia para que estas mulheres ganhem força interior, consigam controlar suas relações e tudo que está à sua volta, 
assim como defender seus direitos. É a partir dele, portanto, que as mulheres podem estabelecer, durante o período parturitivo, um poder de decisão sobre todas as dimensões vivenciadas (Mouta, Silva, Melo, Lopes \& Moreira, 2017).

\section{Considerações Finais}

Passados aproximadamente 16 anos da implantação de políticas de saúde como a da Política Nacional de Humanização e a Política Nacional de Atenção Integral à Saúde da Mulher, a realidade da violência obstétrica persiste nas maternidades brasileiras.

Segundo os relatos das parturientes, as situações acima discutidas ocorrem rotineiramente, com diversas formas de violência obstétrica, além do uso de práticas obstétricas consideradas prejudiciais, ineficazes, sem evidências ou realizadas de forma inadequada, ou seja, contrárias às recomendações da OMS na atenção ao parto.

A identificação de diferentes fatores críticos demonstra que existem falhas significativas nos processos de cuidado na assistência à mulher, evidenciando o desrespeito aos seus direitos. Estes fatores são, em sua maioria, opostos ao que a OMS preconiza, portanto, a pesquisa aponta ser necessário que no pré-natal essas mulheres sejam mais esclarecidas quanto às possibilidades de um parto seguro, livre de danos, procedimentos desnecessários, violência verbal e física. Nesse sentido, é preciso criar espaços de empoderamento feminino para a não ocorrência de procedimentos e atitudes caracterizados como violência obstétrica. Desta forma, este tema deve estar entre os conteúdos que a população deve tomar conhecimento e discutir como pauta para a defesa dos direitos femininos.

Esta pesquisa também propõe a necessidade de mais discussões e aprimoramento dos profissionais envolvidos no trabalho de pré-parto e parto, por meio de cursos de graduação, residência ou pós-graduação, em busca, principalmente, de valorizar a humanização no cuidado obstétrico. É preciso criar espaços de sensibilização desses profissionais e suas instituições para a mudança de condutas.

Como um limite do estudo, pode-se considerar que não é possível afirmar que outras violências não foram sofridas, isso devido à dificuldade da percepção da violência, principalmente pela desapropriação da mulher do seu processo de parir e de seus direitos como mulher, usuária do SUS, mãe e cidadã.

\section{Referências}

Agência Nacional de Vigilância Sanitária [ANVISA]. (2017) Medidas de Prevenção e Critérios Diagnósticos de Infecções Puerperais em Parto Vaginal e Cirurgia Cesariana. Agência Nacional de Vigilância Sanitária. Brasília: Anvisa. https://doi.org/10.1590/S1414-32832010005000035.

Aguiar, J. M. \& D'Oliveira, A. F. P. L. (2011). Violência institucional em maternidades públicas sob a ótica das usuárias. Interface (Botucatu), 15(36), 79-92.

Andrade, P.D., Silva, J., Diniz, C., \& Caminha, M. D. (2016). Fatores associados à violência obstétrica na assistência ao parto vaginal em uma maternidade de alta complexidade em Recife, Pernambuco. Rev. bras. saúde matern. Infant, 16(1), 29-37. https://doi.org/10.1590/1806-93042016000100004.

Assis, J. F. (2018). Interseccionalidade, racismo institucional e direitos humanos: compreensões à violência obstétrica. Serv. Soc. Soc [Internet], 133, 547-565. http://dx.doi.org/10.1590/0101-6628.159.

Bardin, L. (2011). Análise de conteúdo. (4a ed.), Edições.

Biscegli, T. S, Grio, J. M., Melles, L. C., Ribeiro, S. R. \& Gonsaga, R. A. T. (2015). Violência obstétrica: perfil assistencial de uma maternidade escola do interior do estado de São Paulo. CuidArte, Enferm., 9(1), 18-25.

Bohren, M. A., Hunter, E. C., Munthe-Kaas, H. M., Souza, J. P., Vogel, J. P. \& Gulmezoglu, A. M. (2014). Facilitators and barriers to facility-based delivery in low- and middle-income countries: a qualitative evidence synthesis. Reprod Health, 11(1),71. https://doi.org/10.1186/1742-4755-11-71.

Brasil. (2003). Humaniza SUS: política nacional de humanização. Brasília: Ministério da Saúde. http://bvsms.saude.gov.br/bvs/publicacoes/humanizaSus.pdf

Brasil. (2004). Política nacional de atenção integral à saúde da mulher: princípios e diretrizes. Ministério da Saúde, Secretaria de Atenção à Saúde, Departamento de Ações Programáticas Estratégicas. https://conselho.saude.gov.br/ultimas_noticias/2007/politica_mulher.pdf

Castro, T. A. D. V. (2020). Direito ao acompanhante, violência obstétrica e poder familiar. Pensar, Fortaleza, 25(14), 1-12. https://doi.org/10.5020/23172150.2020.10093. 
Chadwick, R. J., Cooper, D.\& Harries, J. (2014). Narratives of distress about birth in South African public maternity settings: a qualitative study. Midwifery 30(7), 862-868. https://doi.org/10.1016/j.midw.2013.12.014.

Diniz, S., Salgado, H., Andrezzo, H., Carvalho, P., Carvalho, P., Aguiar, C. \& Niy, D. (2015). Violência obstétrica como questão para a saúde pública no Brasil: origens, definições, tipologia, impactos sobre a saúde materna, e propostas para sua prevenção. Journal of Human Growth and Development, 25(3), 377-384. https://dx.doi.org/10.7322/jhgd.106080

Gabriel, A. B. \& Santos, B. R. G. (2020). A Injustiça Epistêmica na violência obstétrica. Rev. Estud. Fem, 28(2), e60012. https://doi.org/10.1590/1806-95842020v28n260012.

Giacomini, S. M. \& Hirsch, O. N. (2020). Natural" and/or "Humanized" Childbirth? A Reflection from the Class Perspective. Rev. Estud. Fem., 28(1), e57704. https://doi.org/10.1590/1806-9584-2020v28n157704.

Jardim, D. M. B., \& Modena, C. M. (2019). A violência obstétrica no cotidiano assistencial e suas características. Revista Latino-Americana de Enfermagem, 26, e3069. https://doi.org/10.1590/1518-8345.2450.3069

Katz, L., Amorim, M. M., Giordano, J. C., Bastos, M. H. \& Brilhante, A. V. M. (2020) Who is afraid of obstetric violence? Rev. Bras. Saude Mater. Infant. [Internet], 20(2), 623-626. https://doi.org/10.1590/1806-93042020000200017.

Lansky, S., Souza, K. V., Peixoto, E. R. M., Oliveira, B. J., Diniz, C. S. G., Vieira, N. F., Cunha, R. O. \& Friche, A. A. L. (2019). Obstetric violence: influences of the Senses of Birth exhibition in pregnant women childbirth experience. Ciênc. saúde coletiva [Internet], 24(8), 2811-2823. https://doi.org/10.1590/1413-81232018248.30102017.

Mouta, R. J. O., Silva, T. M. A., Melo, P. T. S., Lopes, N. S. \& Moreira, V. A. (2017). Birth plan as a female empowerment strategy. Rev baiana enferm. [Internet], 31(4), e20275. http://dx.doi.org/10.18471/rbe.v31i4.20275.

Moyer, C. A., Adongo, P. B., Aborigo, R. A., Hodgson, A \& Engmann, C. M. (2014) 'They treat you like you are not a human being': maltreatment during labour and delivery in rural northern Ghana. Midwifery, 30(2), 262-8. https://doi.org/10.1016/j.midw.2013.05.006.

Oliveira, T. R., Costa, R. E. O. L., Monte, N. L., Veras, J. M. M. F. \& Sá, M. I. M. R. (2017). Percepção das mulheres sobre violência obstétrica. Rev enferm UFPE On line. [Internet], 11(1), 40-6. https://doi.org/10.5205/reuol.9978-88449-6-1101201701.

Organização Mundial da Saúde [OMS]. (1996). Tecnologia apropriada para partos e nascimentos. Recomendações da Organização Mundial de Saúde. Maternidade Segura. Assistência ao parto normal: um guia prático. Genebra: WHO.

Organização Mundial da Saúde [OMS]. (2014). The prevention and elimination of disrespect and abuse during facility-nbased chidlbirth. https://apps.who.int/iris/bitstream/handle/10665/134588/WHO_RHR_14.23_eng.pdf;jsessionid=36ACDA15E05ADD66688F46B7E783442F?sequence=1.

Porto, A. M. F., Amorim, M. M. R. \& Souza, A. S. R. (2010). Assistência ao primeiro período do trabalho de parto baseada em evidências. Femina, 38 (10), 527-37.

Reis, T. L. R., Padoin, S. M. M., Toebe, T. R., Pasa, P, C. C. \& Quadros, J. S. (2017). Autonomia feminina no processo de parto e nascimento: revisão integrativa da literatura. Rev. Gaúcha Enferm. [Internet], 38(1), e64677. https://doi.org/10.1590/1983-1447.2017.01.64677.

Rocha, N. F. F. \& Ferreira, J. (2020). A escolha da via de parto e a autonomia das mulheres no Brasil: uma revisão integrativa. Saúde debate [Internet], 44(125), 556-568. https://doi.org/10.1590/0103-1104202012521.

Rodrigues, D. P, Alves, V. H., Penna, L. H. G., Branco, M. B. L. R., Souza, R. M. P. \& Padoin, S. M. M. (2014). A violência na assistência obstétrica no processo parturitivo: um estudo descritivo-exploratório. Brazilian Journal of Nursing, 13, 399-401.

Sampaio, J., Tavares, T. L. A. \& Herculano, T. B. (2019). Um corte na alma: como parturientes e doulas significam a violência obstétrica que experienciam. Rev. Estud. Fem, 27(3), e56406. https://doi.org/10.1590/1806-9584-2019v27n356406.

Schettini, N. J. C., Griboski, R. A. \& Faustino, A. M. (2017). Partos normais assistidos por enfermeiras obstétricas: posição materna e a relação com lacerações perineais espontâneas. Rev Enferm UFPE On Line, 11(2), 932-40. https://periodicos.ufpe.br/revistas/revistaenfermagem/article/view/13462

Sena, L. M. \& Tesser, C.D. (2017). Violência obstétrica no Brasil e o ciberativismo de mulheres mães: relato de duas experiências. Interface (Botucatu) [Internet], 21(60), 209-220. https://doi.org/10.1590/1807-57622015.0896.

Tesser, C. D., Knobel, R., Andrezzo, H. F. de A., \& Diniz, S. G. (2015). Violência obstétrica e prevenção quaternária: o que é e o que fazer. Revista Brasileira de Medicina de Família e Comunidade, 10(35), 1-12. https://doi.org/10.5712/rbmfc10(35)1013. 